\title{
3D Modeling of the Magnetization of Superconducting Rectangular-Based Bulks and Tape Stacks
}

\author{
M. Kapolka, V. M. R. Zermeño, S. Zou, A. Morandi, P. L. Ribani, E. Pardo, F. Grilli
}

\begin{abstract}
In recent years, numerical models have become popular and powerful tools to investigate the electromagnetic behavior of superconductors. One domain where this advances are most necessary is the 3D modeling of the electromagnetic behavior of superconductors. For this purpose, a benchmark problem consisting of superconducting cube subjected to an AC magnetic field perpendicular to one of its faces has been recently defined and successfully solved. In this work, a situation more relevant for applications is investigated: a superconducting parallelepiped bulk with the magnetic field parallel to two of its faces and making an angle with the other one without and with a further constraint on the possible directions of the current. The latter constraint can be used to model the magnetization of a stack of high-temperature superconductor tapes, which are electrically insulated in one direction. For the present study three different numerical approaches are used: the Minimum Electro-Magnetic Entropy Production (MEMEP) method, the $H$ formulation of Maxwell's equations and the Volume Integral Method (VIM) for 3D eddy currents computation. The results in terms of current density profiles and energy dissipation are compared, and the differences in the two situations of unconstrained and constrained current flow are pointed out. In addition, various technical issues related to the 3D modeling of superconductors are discussed and information about the computational effort required by each model is provided. This works constitutes a concrete result of the collaborative effort taking place within the HTS numerical modeling community and will hopefully serve as a stepping stone for future joint investigations.
\end{abstract}

Index Terms-Numerical modeling, magnetization, AC losses, 3D, bulks, stacks of HTS tapes

\section{INTRODUCTION}

Numerical models have become popular tools for investigating the behavior and predict the performance of hightemperature superconductor (HTS) applications [1], [2]. Following this development, dedicated workshops [3] and - more recently - a summer school [4] have been organized. A

Document written on September 19, 2017.

M. Kapolka, E. Pardo are with the Slovak Academy of Sciences, Institute of Electrical Engineering, Bratislava, Slovakia.

V. M. R. Zermeño, S. Zou and F. Grilli are with the Karlsruhe institute of Technology, Institute for Technical Physics, Karlsruhe, Germany.

P. L. Ribani and A. Morandi are with the University of Bologna, Italy.

M. Kapolka and E. Pardo acknowledge the use of computing resources provided by the project SIVVP, ITMS 26230120002 supported by the Research \& Development Operational Programme funded by the ERDF, the financial support of the Grant Agency of the Ministry of Education of the Slovak Republic and the Slovak Academy of Sciences (VEGA) under contract No. 2/0126/15.

Corresponding author's email: francesco.grilli@kit.edu. workgroup and a website have been set up to collect related publications, share model files and propose benchmarks [5].

At the workshops it has been recognized that 2D modeling of superconductors has reached a mature status of development, with models able to handle the very large numbers of conductors in coils and magnets, and very detailed material properties, including, for example, complex angular dependencies of the critical current density on the magnetic field. On the other hand, 3D modeling is not very widely diffused in the applied superconductivity community: several numerical approaches have been proposed and used to solve relatively simple problems [6], [7], [8], [9], [10], but without the accuracy, benchmarking and validation with experimental data typical of their 2D counterparts.

In order to support the development and validation of efficient 3D model, a benchmark has been set: the simulation of a cube of an HTS cube subjected to an AC magnetic field. This is a situation for which analytical solutions do not exist and cannot be simplified to a 2D problem. Recent work by Pardo and Kapolka with the Minimum Electro-Magnetic Entropy Production (MEMEP) method has shown, for a homogeneous and isotropic superconductor, the existence of current paths outside the planes perpendicular to the applied field [11], [12]. The benchmark has been solved by three different numerical approaches, and the results published on the website [5].

In this contribution, we push forward the 3D numerical modeling and investigate the magnetization of a superconducting parallelepiped subjected to an AC magnetic field making an angle with the normal to the larger surface, as shown in Fig. 1. This is a situation of interest because, in addition to be a fully 3D situation for which no analytical solutions exist, it represents a configuration similar to that found in magnetization measurements of superconducting samples by VSM or SQUID magnetometers.

In addition to a homogeneous and isotropic superconductor, we also study the case where no current can flow in the $z$ direction. This condition can be used to model different realistic cases: for example, a bulk ceramic HTS material with the $c$ axis parallel to $z$, in which, due to the strong anisotropy, no substantial current circulates in the $z$ direction. Another case of practical interest is that of stacks of HTS coated conductors used as permanent magnets in superconducting motor applications [13], where the current cannot flow along $z$ due to the presence of high-resistivity layers in the stack.

For this investigation, we use different numerical approaches: in addition to the already mentioned MEMEP 


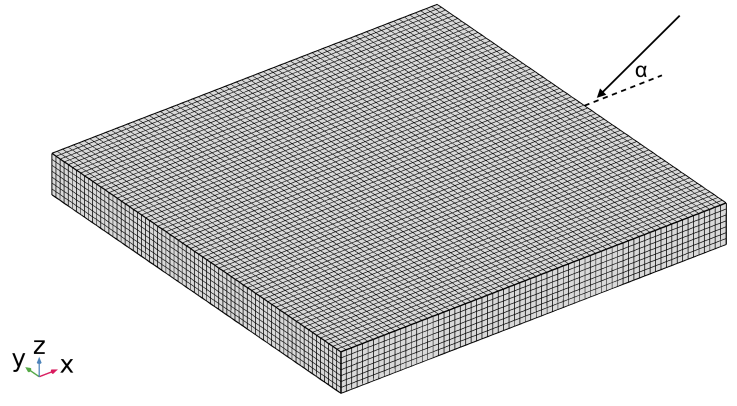

Fig. 1. Geometry of the parallelepiped under study. The external AC magnetic field is applied in the $x z$ plane, at $30^{\circ}$ with respect to the $x$ axis. The volume is discretized in $71 \times 71 \times 7$ cells.

method, the $H$-formulation of Maxwell's equations and the Volume Integral Method (VIEM) for 3D eddy currents computation.

\section{Problem Definition}

The problem under investigation is that of a superconducting parallelepiped, with a square base of side $w=10 \mathrm{~mm}$ and height $1 \mathrm{~mm}$, as represented in Fig. 11. The parallelepiped is subjected to a uniform external sinusoidal magnetic field in the $x z$ plane at an angle $\alpha=30^{\circ}$ with respect to the $x$ axis, of amplitude $B_{\mathrm{a}}=200 \mathrm{mT}$ and frequency $f=50 \mathrm{~Hz}$. The superconductor is modeled as a material with non-linear resistivity

$$
\rho(J)=\frac{E}{E_{\mathrm{c}}}\left|\frac{J}{J_{\mathrm{c}}}\right|^{n-1},
$$

where $J$ and $E$ are the moduli of the current density and of the electric field, respectively, $E_{\mathrm{c}}=1 \times 10^{-4} \mathrm{~V} \mathrm{~m}^{-1}, J_{\mathrm{c}}=$ $1 \times 10^{8} \mathrm{~A} \mathrm{~m}^{-2}, n=25$. Under the assumption of isotropic medium, the directions of the electric field and of the current density are the same.

In addition to an isotropic resistivity (corresponding to a homogeneous and isotropic superconducting bulk), the situation where the current cannot flow in the $z$ direction is also considered. This constraint on the direction of the current flow can be used to model the magnetization of stacks of HTS coated conductors as a homogeneous bulk [14]. For brevity, the two situations will be refereed to as bulk and stack, respectively.

The AC loss (per cycle) is calculated in two different ways [15]:

- by integrating the instantaneous power dissipation $\mathbf{J} \cdot \mathbf{E}$ in the superconductor

$$
Q_{\mathrm{JE}}=2 \int_{1 / 2 f}^{1 / f} \int_{\Omega} \mathbf{J} \cdot \mathbf{E} \mathrm{d} \Omega \mathrm{d} t,
$$

where $\Omega$ is the superconducting volume and the time interval is taken after half AC cycle;

- by integrating the magnetization loop

$$
Q_{\mathrm{MH}}=-\mu_{0} \oint m_{\mathrm{a}} \mathrm{d} H_{\mathrm{a}}
$$

where $m_{\mathrm{a}}$ is the magnetic moment in the direction of the applied field $H_{\mathrm{a}}$.

\section{DESCRIPTION OF THE NUMERICAL MODELS}

The Minimum Electromagnetic Entropy Production (MEMEP) model is based on a variational method. The method minimizes a certain functional [12], which uses the effective magnetization $\mathbf{T}$. The $\mathbf{T}$ vector is zero outside the sample, and hence the method discretizes the geometry of only the sample, which reduces the degrees of freedom and computing time. The mesh is split to three sets of sectors, which are overlapped by $1 / 3$ of sector size. The use of sectors further reduces the computing time and prepares the program for highly efficient parallel calculation. The program is written in $\mathrm{C}++$ and uses protocols like OpenMP and BoostMPI for parallel calculation in computer clusters.

The FEM model is based on the $H$-formulation of Maxwell's equations implemented in the finite-element software package Comsol Multiphysics [16], [17], [18]. The superconducting parallelepiped is surrounded by a cubic air domain (side $100 \mathrm{~mm}$ ), on whose faces the external magnetic field is applied as a Dirichlet boundary condition. The air is modeled as a material with very large electrical resistivity $(1 \Omega \mathrm{m})$. In the case of the homogenized stack, the superconductor is assigned an anisotropic resistivity, the power-law resistivity of (1) in the $x$ and $y$ direction, and $\rho=1 \Omega \mathrm{m}$ in the $z$ direction, so that the current cannot flow along $z$. The superconducting and air domains are meshed with hexahedral and tetrahedral elements, respectively. A "jacket" of prism elements has to be created around the superconducting domain, in order to join the other two types of mesh elements [14].

The volume integral equation method (VIEM) is based on the $\mathbf{A}-\phi$ formulation of the eddy current problem and is implemented by means of a homemade computer program written in FORTRAN90. The superconductor domain is subdivided in a finite number of hexahedral elements. No discretization of the surrounding air is needed. Edge elements shape functions are used for relating the current density of each element to the currents through its faces [19]. A weak solution is obtained by taking the loop integrals of the electric field, which allows eliminating the scalar electric potentials from the set of the unknowns. In essence, a circuit is associated to the superconductor domain which contains as many nodes as the number of elements and as many branches as the faces of the mesh noy lying on the boundary. The unknowns of the problem are the loop currents of the circuit. As the current of each element contributes to the magnetic vector potential at any point, a dense interaction matrix is obtained, as inherent for integral methods. Details of the model can be found in [20], [21].

\section{RESults}

This section focuses on the comparison of current density distributions and AC losses in the case of bulk and the stack.

The instantaneous power loss during the first $25 \mathrm{~ms}$ (rise to the peak and first cycle of the applied field) is shown in Fig. 2 The total energy loss during one cycle (calculated in the time interval from peak to peak of the applied field) are reported in Table I]. The results obtained with the different models are in excellent agreement with each other. This happens for both 
methods used to calculate the losses, given by equations (2) and (3). The agreement between the models is consistent with the previously reported results found for the benchmark case of a cube subjected to an $\mathrm{AC}$ field perpendicular to one of its faces, which are fully documented in [5] (Benchmark \#5).

TABLE I

COMPARISON OF AC LOSSES (IN MJ) IN THE BULK AND IN THE STACK, CALCULATED WITH TWO DIFFERENT METHODS.

\begin{tabular}{ccccc} 
& $Q_{\mathrm{JE}}$ bulk & $Q_{\mathrm{MH}}$ bulk & $Q_{\mathrm{JE}}$ stack & $Q_{\mathrm{MH}}$ stack \\
\hline MEMEP & 4.58 & 4.62 & 3.48 & 3.50 \\
$H$-formulation & 4.59 & 4.62 & 3.47 & 3.45 \\
VIEM & 4.67 & 4.70 & 3.56 & 3.56
\end{tabular}

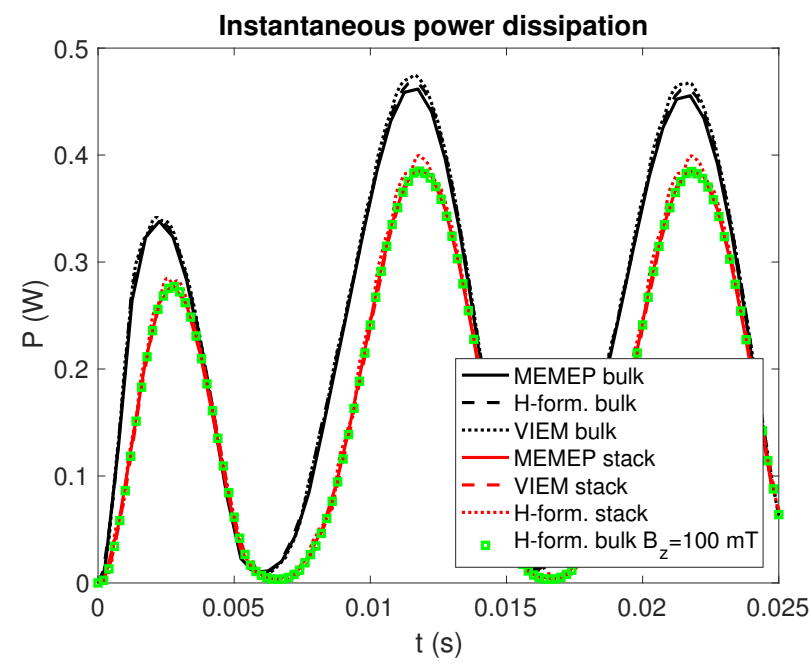

Fig. 2. Instantaneous power dissipation in the bulk (black) and stack (red), calculated with the different models: MEMEP (continuous line), $\mathrm{H}$ formulation (dashed line) and VIEM (dotted line). The results for a bulk subjected to a field of $100 \mathrm{mT}$ (calculated with the $H$-formulation) is also shown.

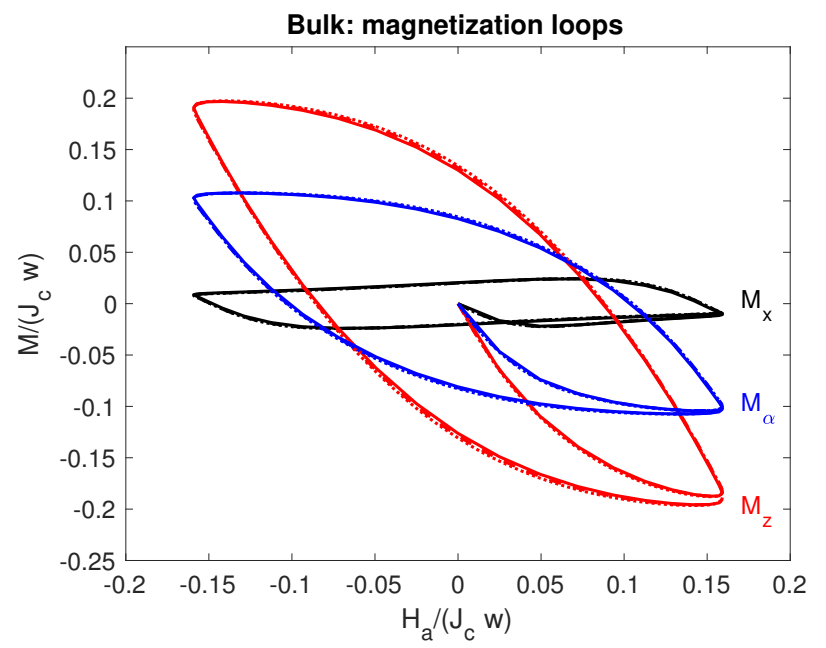

Fig. 3. Magnetization loops of the bulk: the magnetization is calculated along $x$ (black), $z$ (red) and the direction of the field (blue). Each magnetization curve is calculated with the three models: MEMEP (continuous line), $\mathrm{H}$ formulation (dashed line) and VIEM (dotted line). The results of the different models perfectly overlap.

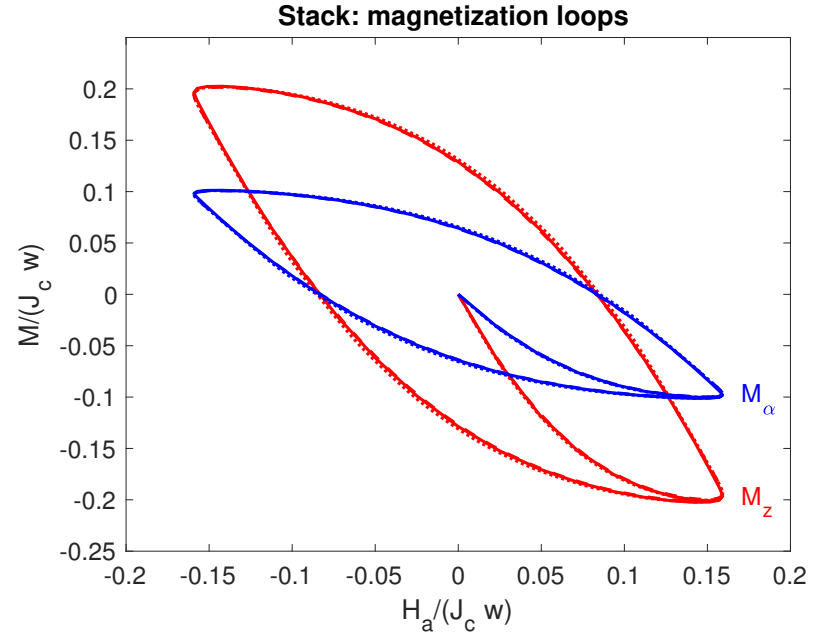

Fig. 4. Magnetization loops of the stack. The magnetization is calculated along $z$ (red) and the direction of the field (blue). Each magnetization curve is calculated with the three models: MEMEP (continuous line), $H$-formulation (dashed line) and VIEM (dotted line). The results of the different models perfectly overlap.

Figures 3 and 4 show the magnetization loops in the bulk and the stack respectively. The $x$ and $z$ components of the magnetization are shown along with the component along the magnetic field direction $M_{\alpha}=M_{x} \cos \alpha+M_{y} \sin \alpha$. The data shown are obtained by the ration between the calculated total magnetic moment and the volume of the superconductor domain, normalized with respect to $J_{\mathrm{c}} w$ (with $w$ being the side of the base of the parallelepiped). The applied magnetic field appearing on the abscissae is normalized by $J_{\mathrm{c}} w$ as well, so that both quantities plotted in the graph are non-dimensional.

Figures 2,3 and 4 indicate that the stack has lower losses than the bulk. This is because a lower total shielding current is induced in the stack. In particular, in the stack case, the $x$ component of the magnetic field cannot induce current loops in the $y z$ plane, so the situation is similar to having just a field of amplitude $200 \mathrm{mT} \times \sin \alpha=100 \mathrm{mT}$ directed along $z$. This is confirmed by the curves of instantaneous power dissipation in Fig. 2

In the stack, the fact of having the constraint that no current can flow in the $z$ direction leads to very different current density distributions than in the case of the bulk. All the current density distributions that follow are taken at the instant of the peak of the applied field, $t=5 \mathrm{~ms}$.

Fig. 5 shows that, in the bulk, the pattern of the $J_{x}$ component is influenced by the angle of the incident magnetic field: with the exception of the mid-plane (the third of the five displayed), the $J_{x}$ distribution varies along $z$. In the stack, the $J_{x}$ distribution is perfectly symmetric. In the mid-plane, it resembles the current distribution of a stack of long tapes subjected to a field perpendicular to the flat face of the tapes. One can compare this, for example, to the 2D results shown in Fig. 2 of [22] or Fig. 6 of [23].

The differences between bulks and tapes are even more striking by looking at the $J_{y}$ component (Fig. 66. At the midplane of the bulk, the cross-section is completely saturated with positive and negative currents, because the field is large 

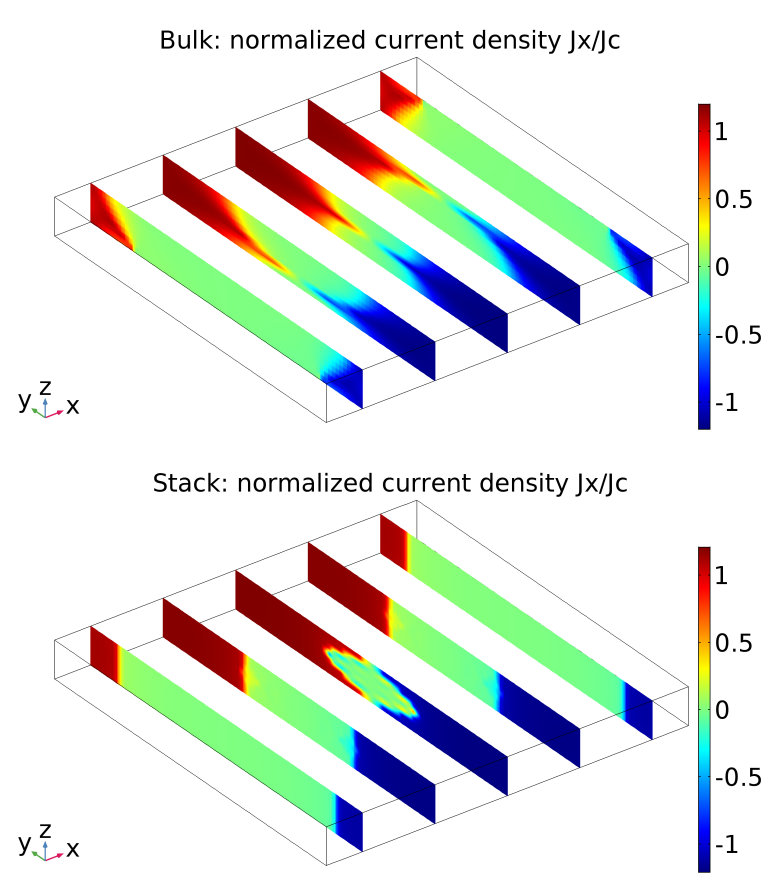

Fig. 5. Distribution of $J_{x}$ on five $y z$ cross-sections of the bulk (top) and stack (bottom).

enough to fully penetrate the bulk. The line of separation between positive and negative currents is oriented along the direction of the applied field. At the mid-plane of the stack, however, the situation is completely different. The distribution is symmetric (in fact, it is identical to the $J_{x}$ distribution discussed above): in the stack the current cannot flow along $z$, and so only the field directed along $z$ can induce current in the the superconductor. Interestingly, the amplitude of the $z$ component is only half of that of the applied field ( $\sin \alpha=0.5)$, and it is not sufficient to fully penetrate the superconductor (as, on the contrary, was the case with the bulk). As a consequence, a current-free core remains.

A more detailed view of the $J_{y}$ distribution in the bulk is given in Fig. 7, which shows the profiles along three particular lines on the plane $z=0$ : the excellent agreement between the models is demonstrated not only for integral quantities like $\mathrm{AC}$ loss and magnetization, but also for local current density profiles.

Finally, Fig. 8 shows the $J_{z}$ component in the bulk on the plane $z=0$. With the exception of some small concentrations in the corners, these currents are small (about $30 \%$ of $J_{\mathrm{c}}$ at most), but not negligible.

Table III lists typical computation times for simulating the two problems (1.25 AC cycles) with the different models and two different discretizations of the superconducting volume. The MEMEP method can take good advantage of parallelization. On a 6-node cluster, the computing times for the $71 \times 71 \times 7$ discretization of the bulk and stack reduce to 2.5 and 1 day, respectively.
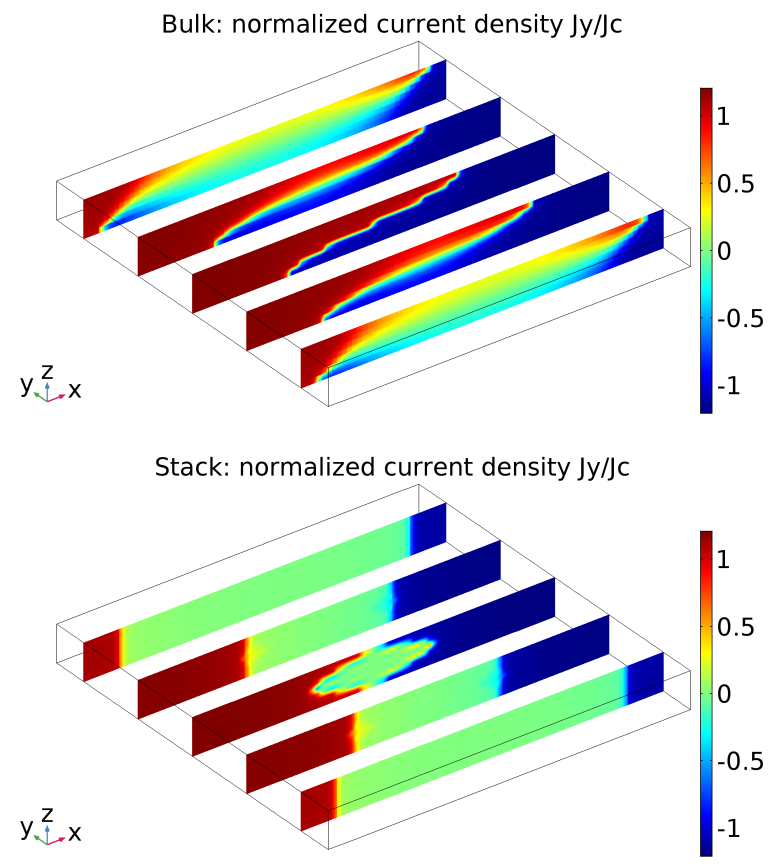

Fig. 6. Distribution of $J_{y}$ on five $x z$ cross-sections of the bulk (top) and stack (bottom).

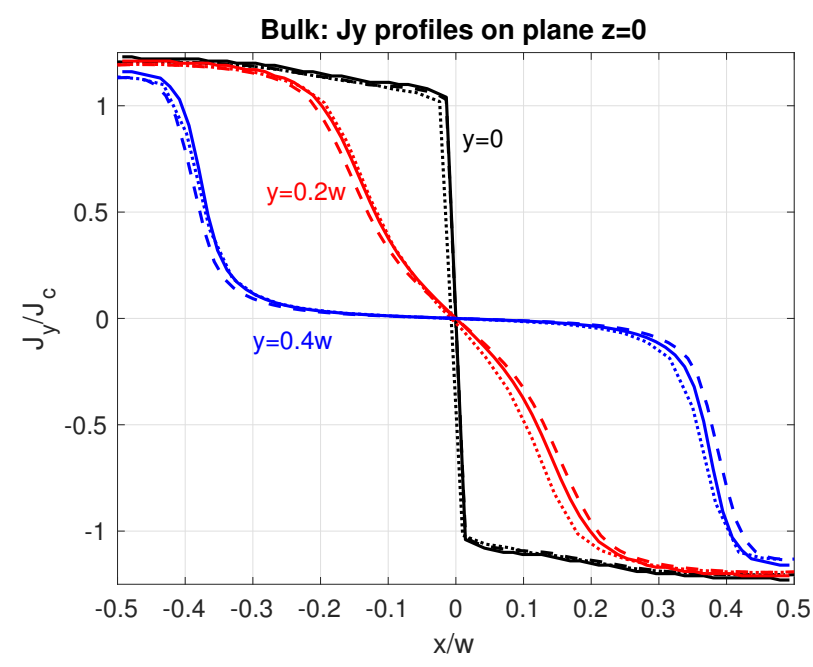

Fig. 7. Distribution of $J_{y}$ along three lines on the $z=0$ plane, calculated with the three models: MEMEP (continuous line), $H$-formulation (dashed line) and VIEM (dotted line).

\section{CONCLUSION}

This work presented the investigation of the magnetization of superconducting rectangular-based bulks and tape stacks by $3 \mathrm{D}$ numerical simulations. Three different numerical approaches were compared and an excellent agreement between them was found. The magnetization loops, the AC loss and the current patterns caused by an external magnetic field making an angle with the normal to the larger surface of the geometries under study are different in the case of bulks and stacks, due to the fact that in the stack geometry the current cannot flow in the direction perpendicular to the faces of the tapes. The computation times strongly depend on the utilized geometry 


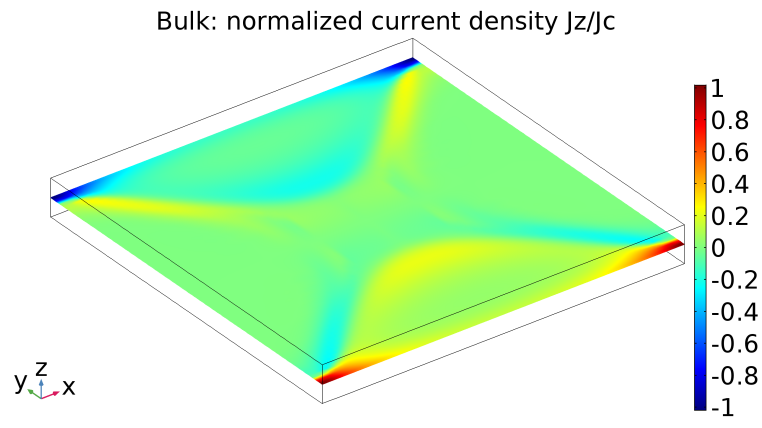

Fig. 8. Distribution of $J_{z}$ on the plane $z=0$ of the bulk.

TABLE II

COMPUTATION TIMES FOR BULK AND STACKS FOR DIFFERENT GEOMETRY DISCRETIZATIONS.

\begin{tabular}{|c|c|c|}
\hline Bulk & $24 \times 24 \times 8$ & $71 \times 71 \times 7$ \\
\hline MEMEP $^{1}$ & $8 \mathrm{~h}$ & $7.7 \mathrm{~d}$ \\
\hline$H$-formulation ${ }^{2}$ & $13 \mathrm{~h}$ & $4.2 \mathrm{~d}$ \\
\hline VIM $^{3}$ & $23 \mathrm{~h}$ & - \\
\hline Stack & $24 \times 24 \times 8$ & $71 \times 7 \times 7$ \\
\hline MEMEP $^{1}$ & $2.2 \mathrm{~h}$ & $6.0 \mathrm{~d}$ \\
\hline$H$-formulation ${ }^{2}$ & $15 \mathrm{~h}$ & $4.1 \mathrm{~d}$ \\
\hline VIEM $^{3}$ & $9 \mathrm{~h}$ & - \\
\hline \\
\hline & & \\
\hline${ }^{2}$ Intel $^{\circledR}$ Core $^{\mathrm{TM}}$ i7-4960 CPU 64 GB RAM OS: Windows 7 & 5-3570 CPU 8 & OS: Windows 10 \\
\hline
\end{tabular}

discretization. For the considered problem, a discretization of less than 5000 cells is enough to obtain accurate results in the reasonable time of a few hours on standard desktop workstations. This works constitutes a tangible result of the collaborative effort taking place within the HTS numerical modeling community and will hopefully serve as a stepping stone for future joint investigations.

\section{REFERENCES}

[1] F. Sirois and F. Grilli, "Potential and limits of numerical modeling for supporting the development of HTS devices," Superconductor Science and Technology, vol. 28, no. 4, p. 043002, 2015. [Online]. Available: http://dx.doi.org/10.1088/0953-2048/28/4/043002

[2] F. Grilli, "Numerical Modeling of HTS Applications," IEEE Transactions on Applied Superconductivity, vol. 26, no. 3, p. 0500408, 2016. [Online]. Available: http://dx.doi.org/10.1109/TASC.2016.2520083

[3] "Fifth International Worshop on Numerical Modelling of HTS, Bologna, Italy, 15-17 June 2016." [Online]. Available: https://events.unibo.it/ htsmodelling2016/

[4] "First School on Numerical Modeling for Applied Superconductivity, Lausanne, Switzerland 20-24 June 2016," 2016. [Online]. Available: http://supra.epfl.ch/school/

[5] "HTS Modelling Workgroup." [Online]. Available: http://www. htsmodelling.com/

[6] R. Pecher, M. McCulloch, S. J. Chapman, L. Prigozhin, and C. M. Elliott, "3D-modelling of bulk type-II superconductors using unconstrained H-formulation," Institute of Physics Conference Series, vol. 181, 2003.

[7] F. Grilli, M. C. Bouzo, Y. Yang, C. Beduz, and B. Dutoit, "Finite element method analysis of the coupling effect between superconducting filaments of different aspect ratio," Superconductor Science and Technology, vol. 16, no. 10, pp. 1228-1234, 2003. [Online]. Available: http://iopscience.iop.org/0953-2048/16/10/318

[8] A. M. Campbell, "A direct method for obtaining the critical state in two and three dimensions," Superconductor Science and Technology, vol. 22, p. 034005, 2009.
[9] S. Farinon, G. Iannone, P. Fabbricatore, and U. Gambardella, "2D and 3D numerical modeling of experimental magnetization cycles in disks and spheres," Superconductor Science and Technology, vol. 27, no. 10, p. 104005, 2014. [Online]. Available: http: //dx.doi.org/10.1088/0953-2048/27/10/104005

[10] E. Pardo, M. Kapolka, J. Kováč, J. Souc, F. Grilli, and R. N. A. Piqué, "Three-Dimensional Modeling and Measurement of Coupling AC Loss in Soldered Tapes and Striated Coated Conductors," IEEE Transactions on Applied Superconductivity, vol. 26, no. 3, p. 4700607, 2016. [Online]. Available: http://dx.doi.org/doi:10.1109/TASC.2016.2523758

[11] E. Pardo and M. Kapolka, "3D magnetization currents, magnetization loop, and saturation field in superconducting rectangular prisms," Superconductor Science and Technology, vol. 30, no. 6, p. 064007, 2017. [Online]. Available: https://doi.org/10.1088/1361-6668/aa69ed

[12] — , "3D computation of non-linear eddy currents: Variational method and superconducting cubic bulk," Journal of Computational Physics, vol. 344, pp. 339-363, 2017. [Online]. Available: http: //dx.doi.org/10.1016/j.jcp.2017.05.001

[13] A. Patel, S. C. Hopkins, and B. A. Glowacki, "Trapped fields up to 2 $\mathrm{T}$ in a $12 \mathrm{~mm}$ square stack of commercial superconducting tape using pulsed field magnetization," Superconductor Science and Technology, vol. 26, p. 032001, 2013.

[14] S. Zou, "Magnetization of High Temperature Superconducting TrappedField Magnets," Ph.D. dissertation, Karlsruhe Institute of Technology, 2017. [Online]. Available: http://dx.doi.org/10.5445/IR/1000073152

[15] F. Grilli, E. Pardo, A. Stenvall, D. N. Nguyen, W. Yuan, and F. Gömöry, "Computation of Losses in HTS Under the Action of Varying Magnetic Fields and Currents," IEEE Transactions on Applied Superconductivity, vol. 24, no. 1, p. 8200433, 2014. [Online]. Available: http://dx.doi.org/10.1109/TASC.2013.2259827

[16] R. Brambilla, F. Grilli, and L. Martini, "Development of an edge-element model for AC loss computation of high-temperature superconductors," Superconductor Science and Technology, vol. 20, no. 1, pp. 16-24, 2007. [Online]. Available: http://dx.doi.org/10.1088/0953-2048/20/1/004

[17] F. Grilli, R. Brambilla, F. Sirois, A. Stenvall, and S. Memiaghe, "Development of a three-dimensional finite-element model for high-temperature superconductors based on the $H$-formulation," Cryogenics, vol. 53, pp. 142-147, 2013. [Online]. Available: http: //dx.doi.org/10.1016/j.cryogenics.2012.03.007

[18] V. Zermeno, F. Grilli, and F. Sirois, "A full 3-D time-dependent electromagnetic model for Roebel cables," Superconductor Science and Technology, vol. 26, no. 5, p. 052001, 2013. [Online]. Available: http://dx.doi.org/10.1088/0953-2048/26/5/052001

[19] R. Albanese and G. Rubinacci, "Finite Element Methods for the Solution of 3D Eddy Current Problems," Advances in Imaging and Electron Physics, vol. 102, pp. 1 -86, 1997. [Online]. Available: http://dx.doi.org/10.1016/S1076-5670(08)70121-6

[20] A. Cristofolini, M. Fabbri, A. Morandi, F. Negrini, and P. L. Ribani, "Current distribution in a composite superconducting system by means of an equivalent circuit model based on a smooth E-J equivalent material characteristic," Physica C, vol. 372, pp. 1771-1776, 2002. [Online]. Available: http://dx.doi.org/10.1016/S0921-4534(02)01123-1

[21] A. Morandi, "Circuit Methods for three dimensional field analysis in Large Scale Superconducting Systems," Ph.D. dissertation, University of Bologna, Italy, 2004.

[22] F. Grilli, R. Brambilla, and L. Martini, "Modeling High-Temperature Superconducting Tapes by Means of Edge Finite Elements," IEEE Transactions on Applied Superconductivity, vol. 17, no. 2, pp. 31553158, 2007. [Online]. Available: http://dx.doi.org/10.1109/TASC.2007. 902144

[23] E. Pardo, J. Šouc, and J. Kováč, "Ac loss in rebco pancake coils and stacks of them: modelling and measurement," Superconductor Science and Technology, vol. 25, no. 3, p. 035003, 2012. [Online]. Available: stacks.iop.org/SUST/25/035003 